\title{
Ultrafast Time-Resolved Spectroscopy
}

\author{
László Nánai ${ }^{1}$, Szabolcs Beke ${ }^{1}$ and Koji Sugioka ${ }^{2}$ \\ ${ }^{1}$ University of Szeged, Department of General and Environmental Physics \\ Laser Technology Laboratory \\ ${ }^{2}$ RIKEN - The Institute of Physical and Chemical Research, Wako, Saitama, \\ ${ }^{1}$ Hungary \\ ${ }^{2}$ Japan
}

\section{Introduction}

In this chapter we will focus on the subject of spectroscopical investigation of matter (gas, liquid, solid, low dimension materials, etc) using ultrashort (ps, fs) laser pulses in different wavelength region (visible, infrared, $\mathrm{UV}, \mathrm{X}$-ray). We will point out the rigorous difference in aims of classical spectroscopy using highly narrowed electromagnetic waves with high temporal and spatial coherence compared to the wide narrowed spectral structures of ultrashort light pulses.

We will discuss briefly the main characteristics of light-matter interaction on the base of the linear and nonlinear optics assumption. Some outlook of facilities of classical spectroscopy will be made with attempt of time resolved techniques e.g., time of flight and pump and probe techniques. We will also demonstrate some interesting results of ultrafast time resolved spectroscopy in material science and solid state physics.

\section{Elements of linear and nonlinear optics of light-matter interaction}

The study of phenomena induced by laser beam (continuous (CW) and pulsed) on solid surfaces is a widely investigated subject of modern solid state physics and chemistry. From the advent of the first lasers (late 60s) a huge number of scientific papers had been devoted to the investigations of different kinds of laser - matter processes such as laser induced damage, plasma formation, phase transition in different circumstances, micro- and macroprocessing, laser-induced chemical reaction at solid - gas and solid - liquid surfaces etc. The laser became a very useful tool for initiating unique chemical reactions to produce advanced materials (ultra hard ones for example). Number of technological applications, for example laser induced deposition of metals on porous materials and semiconductor surfaces are already in service of high-tech industry (micro- and nanoelectronics). Production with tailored properties of different nanostructures and components serves in chemical industry as catalysators, etc. [1]

Some preliminaries of the light-matter interaction are treated in a quasi-classical approximation:

Semi-classical approximation means that the electromagnetic wave is described classically on the base of Maxwell's equations while the material is described on quantum 
mechanical level (taking into account the electronic, vibration and rotation energy levels of the atoms).

\section{Linear optics approximation}

From Maxwell's equations we get:

$$
\bar{D}=\varepsilon_{0} \bar{E}+\bar{P}(\bar{E}) \quad \text { and } \quad \bar{B}=\mu_{0} \bar{H}+\mu_{0} \bar{M}(\bar{H})
$$

where $\bar{D}$ - electric flux density, $\bar{E}$ - electric field vector, $\bar{B}$ - magnetic flux density, $\bar{H}$ magnetic field vector, $\varepsilon_{0}$ - dielectric constant of vacuum $=8.85 \cdot 10^{-12} \mathrm{C}^{2} \mathrm{~N}^{-1} \mathrm{~m}^{-2}[\mathrm{~F} / \mathrm{m}], \mu_{0}-$ permeability of free space $=4 \pi \cdot 10^{-7} \mathrm{H} / \mathrm{m}$ (we will care materials for which $\bar{M}=0 \rightarrow \bar{B}=\mu_{0} \bar{H}$ ). The polarization

$$
\bar{P}(\bar{E})=\varepsilon_{0} \chi \bar{E}
$$

where $\chi$ - electric susceptibility.

The wave equation for electric field:

$$
\nabla^{2} \bar{E}=\mu_{0} \varepsilon_{0} \frac{\partial^{2} \bar{E}}{\partial t^{2}}+\mu_{0} \varepsilon_{0} \chi \frac{\partial^{2} \bar{E}}{\partial t^{2}}=\mu_{0} \varepsilon_{0} \varepsilon_{r} \frac{\partial^{2} \bar{E}}{\partial t^{2}}
$$

where $\varepsilon_{r}=(1+\chi)$ is the relative dielectric constant.

It is well known from optics that the frequency $v$ of electromagnetic wave and the material refractive index $n$ are related to $c_{0}$ (speed of light in vacuum)

$$
v^{2}=\frac{1}{\mu_{0} \varepsilon_{0}} \frac{1}{\varepsilon_{r}}=\frac{c_{0}^{2}}{\varepsilon_{r}} \quad \text { and } n=\frac{c}{v}
$$

where $c_{0}=3 \cdot 10^{8} \mathrm{~m} / \mathrm{s}$.

The solution of the wave equation can be expressed:

$$
E(z, t)=\operatorname{Re}\{\bar{E}(z, \omega) \exp (-i \bar{k} \bar{r}+i \omega t)\}
$$

where $z$ is the direction of propagation, $\omega=2 \pi v$ the circular frequency and $k$ is the wave vector $(k=2 \pi / \lambda, \lambda=c / v$ the wavelength).

For the materials we have the

- group velocity

$$
v_{g}=\frac{d \omega}{d k}
$$

- phase velocity

$$
v_{p h}=\frac{\omega}{k}=\frac{c}{n}
$$

and the Beer-Lambert law 


$$
I(z)=I_{0} e^{-\alpha z}
$$

where $I_{0}$ is the intensity of the light at the incidence plane, $I(z)$ the intensity in the depth level $\mathrm{z}$, and $\alpha$ is the absorption coefficient.

The real $\left(\varepsilon^{\prime}\right)$ and imaginary $\left(\varepsilon^{\prime \prime}\right)$ parts of dielectric constants at given frequency are related to optical material parameters $n$ and $k$ through Kramers-Kronig relations.

$$
n=\sqrt{\frac{\sqrt{\left(\varepsilon^{\prime}\right)^{2}+\left(\varepsilon^{\prime \prime}\right)^{2}}+\varepsilon^{\prime}}{2}}, \quad k=\sqrt{\frac{\sqrt{\left(\varepsilon^{\prime}\right)^{2}+\left(\varepsilon^{\prime \prime}\right)^{2}}-\varepsilon^{\prime}}{2}} .
$$

The above-mentioned equations describe the light - matter interaction events for relatively low beam intensities where the material constants do not depend on the intensity, but only on the frequency.

\section{Nonlinear optics approximation}

For the laser beam intensities comparable to the electric field strength inside the atoms we have to take into account the higher-order (nonlinear) terms in the field to get the polarization $\bar{P}$. (For $\mathrm{H}$ we got $E \sim 10^{9} \mathrm{~V} / \mathrm{cm}$ and $I \sim 10^{16} \mathrm{~W} / \mathrm{cm}^{2}$ )

$$
\bar{P}(t)=\varepsilon_{0}\left(\chi^{(1)} \bar{E}+\chi^{(2)} \bar{E}^{2}+\chi^{(3)} \bar{E}^{3}+\ldots\right)
$$

where $\chi^{(n)}$ is the $\mathrm{n}^{\text {th }}$ order of the susceptibilities of the medium.

As a result we can get different phenomena e.g., $2^{\text {nd }}$ and higher order harmonic generation, sum- and difference frequency generation, parametric generation, etc.

We note that in all processes the conservation laws for photon energy and momentum (phase matching) should be fulfilled as

$$
\omega=\omega_{1}+\omega_{2}+\omega_{3}+\ldots \text { and } \bar{k}=\bar{k}_{1}+\bar{k}_{2}+\bar{k}_{3}+\ldots
$$

Non-linear optical effects usually called as $\mathrm{N}$ wave mixing processes, where $\mathrm{N}$ is the number of photons participating in reactions. The more photons the weaker the probability of the effect. At very high laser intensities e.g., at very short pulses very high order effects might have been realized. The materials usually have different refractive indices for different frequencies and polarizations; therefore the phase matching for them will be satisfied only for special directions e.g., second harmonics birefringence.

A number of special methods have been developed (due to nonlinear crystals to produce effects such as second harmonic generation (SHG), optical parametric generation (OPG), optical parametric oscillation (OPO), optical parametric amplification (OPA), quick switching features as electrooptical Kerr and Pockel cells [2-4].

\section{The spectroscopy}

\subsection{General remarks}

Spectroscopy in general is the study of the interaction between light and matter such as determination of quantum energy levels in substrates (gas, solid, liquid, etc.). In this "traditional" simple form, one measures the spectroscopic components of the reflected beam 
after transmitted through the medium or emitted from it due to the external excitation of the energetic levels. In a classical his performance one uses - normally - spectrally narrow light beam. This beam may be tuned across discrete energetic levels of the studied target. Different regions of the electromagnetic spectrum provide different kinds of information as a result of the interaction.

The spectroscopic instrument represents - as its main part - a dispersion element (prism or grating with high separation capability of beam wavelengths). Usually this consists also of a slit, light collecting optics, and a detector (see Fig. 1.) called monochromator.

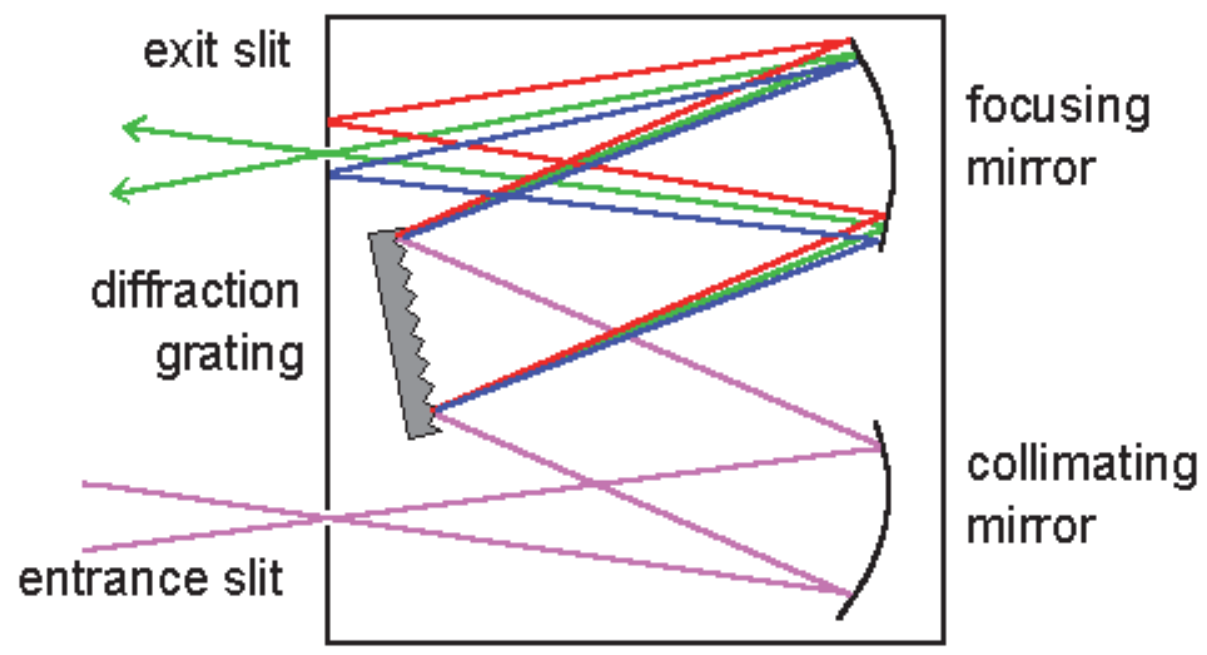

Fig. 1. A general scheme of a monochromator

Depending of the target material structure, composition, and the light-matter interaction type such as direct absorption, transmission, emission of scattering (type Rayleigh, Brillouin, Raman) one can summarize the classical spectroscopy methods as follow [5-11].

- Atomic spectroscopy:

- $\quad$ absorption (AA)

- $\quad$ emission (AES, OES)

- fluorescence (AFS)

- Electron spectroscopy:

- $\quad$ Auger electron spectroscopy (AES)

- $\quad$ X-ray photoelectron spectroscopy (XPS, ESCA)

- Infrared spectroscopy

- molecular spectroscopy

- near infrared absorption spectroscopy (NIR)

- ultraviolet and visible spectroscopy (UV-VIS)

- Nuclear and electron spectroscopy

- $\quad$ electron paramagnetic resonance spectroscopy (EPR, ESR)

- nuclear magnetic resonance spectroscopy (NMR)

- $\quad$ Fourier transform spectroscopy (FT) 
- $\quad$ Laser spectroscopy

- $\quad$ laser-induced fluorescence spectroscopy (LIF)

- Raman spectroscopy (RAMAN)

- resonance ionization spectroscopy (RIS)

- $\quad$ X-ray and $\gamma$-ray spectroscopies

- Mossbauer spectroscopy (MOSSBAUER)

- Newton activation analysis spectroscopy (NAA)

- X-ray fluorescence spectroscopy (XRF)

- $\quad$ extended X-ray absorption fine structure (EXAFS)

and numerous other combined type spectroscopic methods [7-8].

\section{Ultrafast spectroscopy}

Ultrafast spectroscopy is based on using ultrashort laser pulses with pulse duration of ps-fs time region. This technique involves temporally short, therefore spectrally broad light pulses. These kinds of pulses are used to probe directly the dynamics of the system rather than the energy levels themselves. It is very important that the duration of the laser pulses must be shorter than the time scale of the dynamics that one wants to observe.

Taking into account the quantum mechanical considerations we reach the appearance of uncertainty principle e.g., time and energy resolution are related to each other through the Fourier transformation [7].

For Gaussian profile pulses the spectral bandwidth $\Delta v$ of the pulse and its temporal duration $\tau$ on the full width at the maximum level (FWHM) can be written as

$$
\Delta v \tau \geq 2 \ln (2) / \pi
$$

e.g., if $\tau=5$ fs we get $\Delta v=8,8 \cdot 10^{3} \mathrm{~Hz}\left(2940 \mathrm{~cm}^{-1}\right)$.

\section{Ultrafast laser excitation in materials}

\subsection{Impact of laser beam energy to a matter}

Elementary excitations in solids show a complex nonequilibrium behavior. The fastest nonequilibrium processes occur on ultrafast time scales and strongly influence both optical properties and carrier transport. Among condensed phases metals and semiconductors represent an interesting class of practically important targets of nano and microelectronics. During these processes the electronic band structure, optical transition energies, carrier concentration, and phonon frequencies vary over a broad range leading to a variety of ultrafast phenomena. Moreover, the quantum confinement of wave functions in low-dimensional semiconductor nanostructures allows a systematic variation of material properties.

Optical spectroscopy with ultrashort pulses provides direct insight into these processes occurring on a time scale between about $10^{-14}$ and $10^{-10} \mathrm{~s}$.

High amount of laser light energy can be deposited in a very small volume determined by the laser focal spot and penetration depth at a given wavelength. The electromagnetic incident wave will lead to photo-excitation of the electrons due to the large difference of electron and phonon heat capacities $\left(c_{\mathrm{p}}>>c_{\mathrm{e}}\right)$. Therefore in the target material, especially in the case of metals and metal-based nanostructures - one creates a non-equilibrium electron distribution leaving the lattice temperature essentially unchanged $(T \sim 300 \mathrm{~K})$. The rise time 
of non-degenerate electron distribution creation is in the order of a few fs, thus we can say that high temperature non-equilibrium electron distribution has the same rise time as the laser pulse duration. Then over a time scale of a few fs the non-equilibrium electrons redistribute their energy among themselves. It takes place through e.g., electron - electron coulomb interactions resulting in a local equilibrium with temperature $T_{e}$, called the thermalized electron redistribution (with relaxation time $\tau_{e-e}$ ).

The excited thermalized electron gas then transforms the energy through electron - phonon interactions within a relaxation time $\tau_{e-p}$.

Energy transports between electron and phonon subsystems [12-16].

This mass energy transferred to phonon bath will be redistributed among phonons during the relaxation time $\tau_{p-p}$ leading to the equilibrium phonon temperature $T_{l}$.

Therefore we can consider the kinetic evolution of a photo-excited electron - phonon system.

- fast, involving the electron subsystem thermalization (at quite high temperatures) and electron-phonon scattering (at quite moderate temperatures). These are called nonthermal processes. The average time scale is about 1-500 fs.

- slow, involving the phonon-phonon scattering leading to heat conductions, thermal melting and probably ablation (called thermal processes). The average time scale in the case of metals is about 1 ps to a few ns.

In semiconductors and complex nanostructures, the relaxation processes are multistep ones and include different mechanism such as:

Below band gap excitations:

Transitions between electronic states may have different origins, such as:

Transitions from atoms or vacancies,

Transitions from impurity levels into the valence or conduction band continuum,

Transitions (indirect) between excited intraband levels,

Transitions due to the so-called free carrier absorption,

Inter-valence band transitions of holes, and intersubband transitions between valence and conduction subbands

Transitions in low-dimensional semiconductor nanostructures, e.g., quantum wells, wires and dots.

Indirect interband excitations: free carrier absorption due to the presence of free charges in both conduction and valence bands. This requires coupling to a third particle, e.g., a phonon or an impurity because of the conservation laws for wave number vectors.

Inter valence band transitions: due to dipole-allowed transitions of free holes from states in one valence band to states of higher energy in another valence band. For bulk semiconductors with a diamond-like, e.g., silicon and germanium, or zinc-blende lattice like most III-V semiconductors, inter-valence band absorption is dominated by transitions between the heavy hole $(\mathrm{HH})$ and light hole (LH and split-off bands).

Intersubband transitions in quasi-two-dimensional nanostructures: They are characteristics for quantum wells or superlattices in which carrier motion is restricted to a quasi-twodimensional semiconductor layer. Quantum confinement occurs in a situation where the length scale of the potential structure, i.e., the well width, is on the order of the de-Broglie wavelength of the carriers.

Dephasing of coherent polarizations 
Resonant interaction of a coherent ultrashort pulse with a particular transition in the semiconductor creates both a coherent optical polarization between the optically coupled states and carriers (electrons or holes) from energetically lower to higher states in the same or a different band.

With time evaluation this well-defined phase relation is destroyed by a variety of scattering processes which change the relative phase of the wave function between the ground- and excited states. This phase relaxation or so-called dephasing process means a fast decay of the macroscopic polarization and results in a homogeneous broadening of the particular optical transition.

Therefore, the overall excitation-relaxation process could be characterized as it can be seen in Fig. 2.

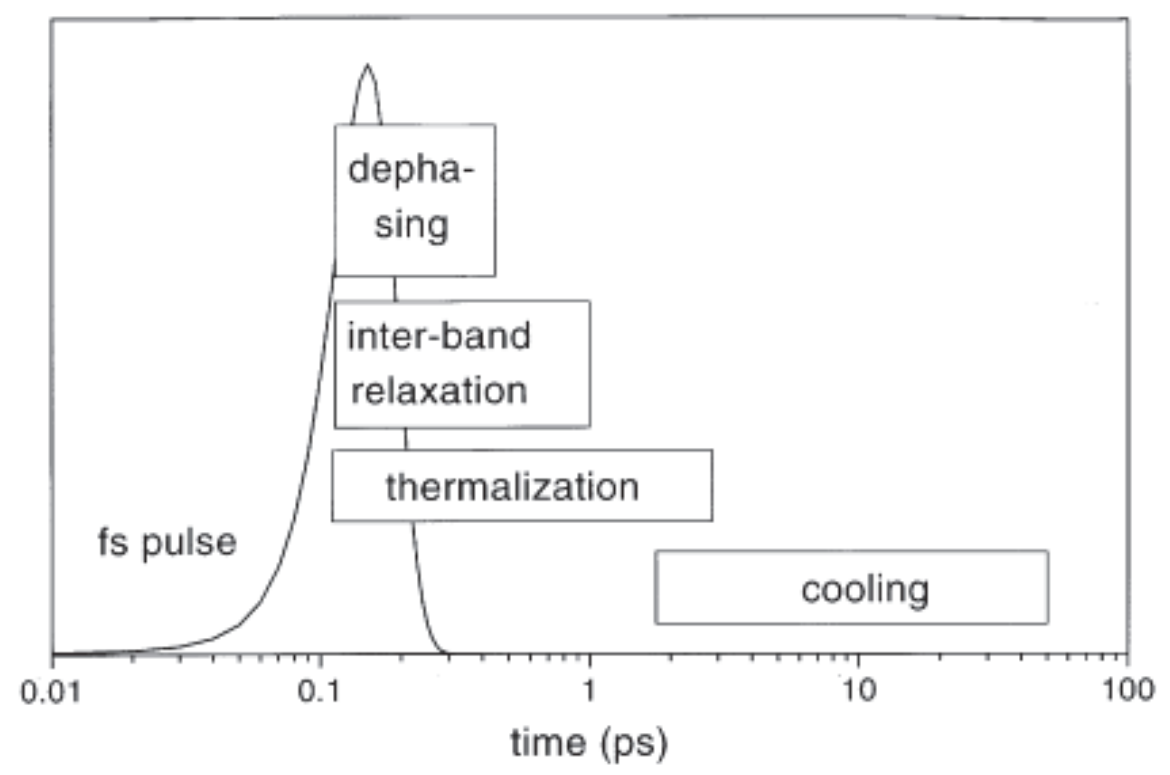

Fig. 2. The scheme of excitation-relaxation processes [12]

\section{Measurements and instrumentations}

As it had been mentioned before the ultrashort laser pulses provide an excellent tools to realize time-resolved experiments with which one can observe transient species in different chemical reactions and follow the dynamical behavior of physical-, chemical- and biological processes. Another important property is that with modest energy, the fs pulses can have huge peak powers. This also makes them suitable for many tasks that we would not normally think of as 'time resolved', including laser ablation of materials, multi-photon absorption (for imaging of biological materials), fragmentation (e.g., DNA into fragments that may be analyzed using mass spectrometry), the conversion to a range of new wavelengths using nonlinear techniques, e.g., infrared light to visible light conversion and 2-photon excited fluorescence, etc. Semiconductor processes and collisions in liquid phase materials are also in the range of a few hundreds of fs [18-20]. 
Direct measurements in fs region are not possible using electrical methods and other nonoptical techniques. The use of specialized photodetectors such as streak cameras or avalanche photodiodes that can resolve picosecond or even 100s of femtoseconds transients in real-time, but are not able to resolve a necessary few fs events, therefore alternative detection techniques are required.

The techniques that are used most frequently are based on the auto- or cross-correlation of two beams of femtosecond pulses. If the target is a nonlinear crystal used for sum-frequency generation, this technique can be used to determine the shape and relative arrival time of two short pulses. If the sample consists absorbing materials normally one uses pump-probe experiments for temporal registrations of events [1,21-30]. Therefore, if we want to measure the dynamics of a fast event, we have to apply a faster tool to do it. Moreover, the use of a not as short as possible laser pulse can induce the shortening transient behavior [31]. The most commonly used scheme of a general pump and probe equipment is sketched in Fig 3. [24]

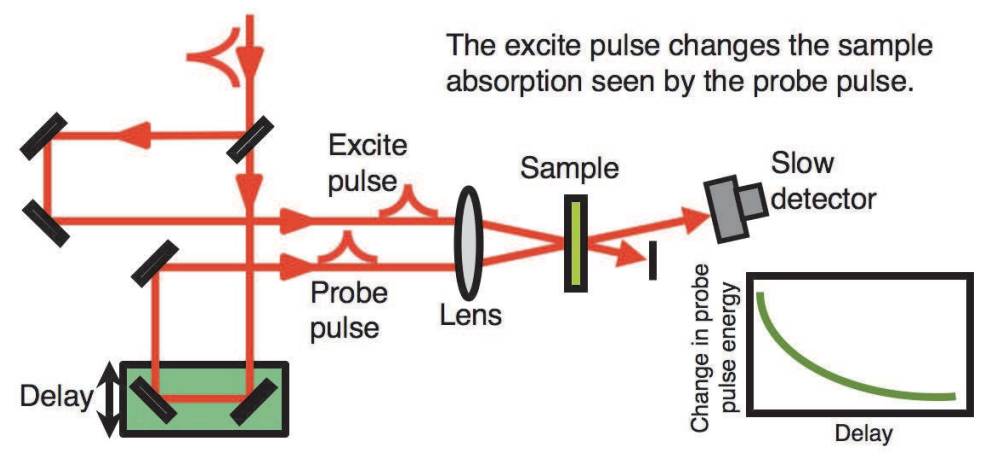

Fig. 3. Schematic of a general pump and probe equipment [24]

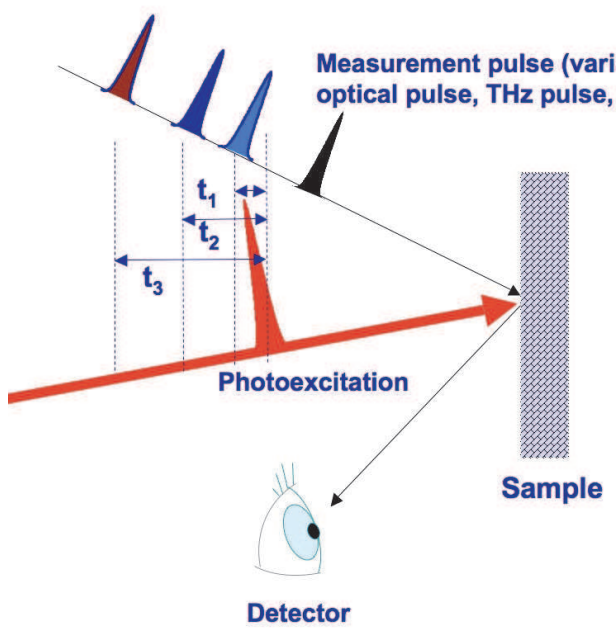

the pulse width $\sim 10 \mathrm{fs}-1 \mathrm{ps}$

Fig. 4. Sketch of pump and probe for different sources 
The delay in the probe arm is usually realized with an optical path enhancement done by a mirror system (Fig 4.).

As we can see the main laser beam is split with a mirror into 2 parts: pump beam with intensity of about $90 \%$ of the original and a probe beam of about $10 \%$ of original. Both pulses are focused upon the target with their spatial overlapping. The delay is realized with variation of a beam path length compared to probe one. The weaker pulse in some of his characteristic (e.g., intensity, polarization, temporal duration) will be modified varying the delay $(\Delta t)$. This is the results of excitation in target material by the pump beam. Repeating the measurements by varying the time delay one determines the temporal dynamics of the excitation.

In some of more sophisticated measurements, one tries to use a focusing object as it can be seen in Fig 5.

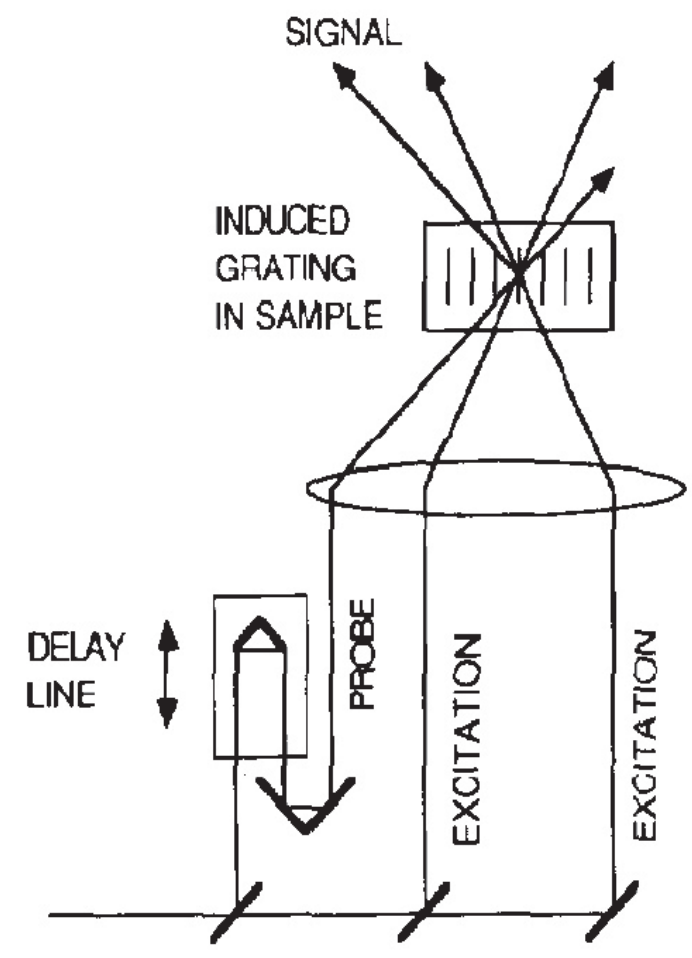

Fig. 5. Schematic of the transient grating experiment. Two excitation pulses are crossed in time and space in the sample. The resultant spatially periodic material excitation is probed by diffraction of a third, variably delayed beam [32].

Different variations of this technique can be used to determine dynamics of events in different fields, such as electron transport in solids, hetero- and nanostructures, induced spin dynamics by magnetic influences etc. Numerous applications had already been developed for chemistry, biology, and life sciences. The resolution achieved by pump and probe method nowadays reaches as hundreds of attoseconds. 


\section{Ultrafast X-ray spectroscopy}

X-rays are very useful tools of modern science as well as solid state physics. The determination of the atomic structures became possible with achievement of coherent X-ray applications. In that frame one uses the static X-ray diffraction technique based on Bragg reflections.

However, the appearance of new pulsed coherent X-ray sources with extremely short pulse duration had opened a way for time dependent investigations. Femtosecond X-ray pulses enable atomic spatial $(\sim 0,1 \mathrm{~mm})$ and high enough temporal resolution to observe the evolution of atomic configurations. In such a way one gets a direct dynamic structural picture [31-35].

Until now, a variety of methods have been developed to generate fs X-ray beams. For example, during the interaction of very high intensity laser pulses with material due to results of electron-atom interaction processes one yields to characteristic brehmstrahlung and line emission. The time duration of $\mathrm{X}$-ray beam generated like as generating fs laser pulse duration, and the energies are in range of $10 \mathrm{eV} \sim 1 \mathrm{MeV}$. Also high intensity coherent $\mathrm{X}$-ray beams may be emerged from laser-produced plasma sources or laser-driven electron $X$-ray sources and synchrotron radiation induced sources [31, 33, 35].

To perform time-resolved measurements in the X-ray regime one can use suitable variants of pump and probe techniques like in optical region (Fig. 6.).

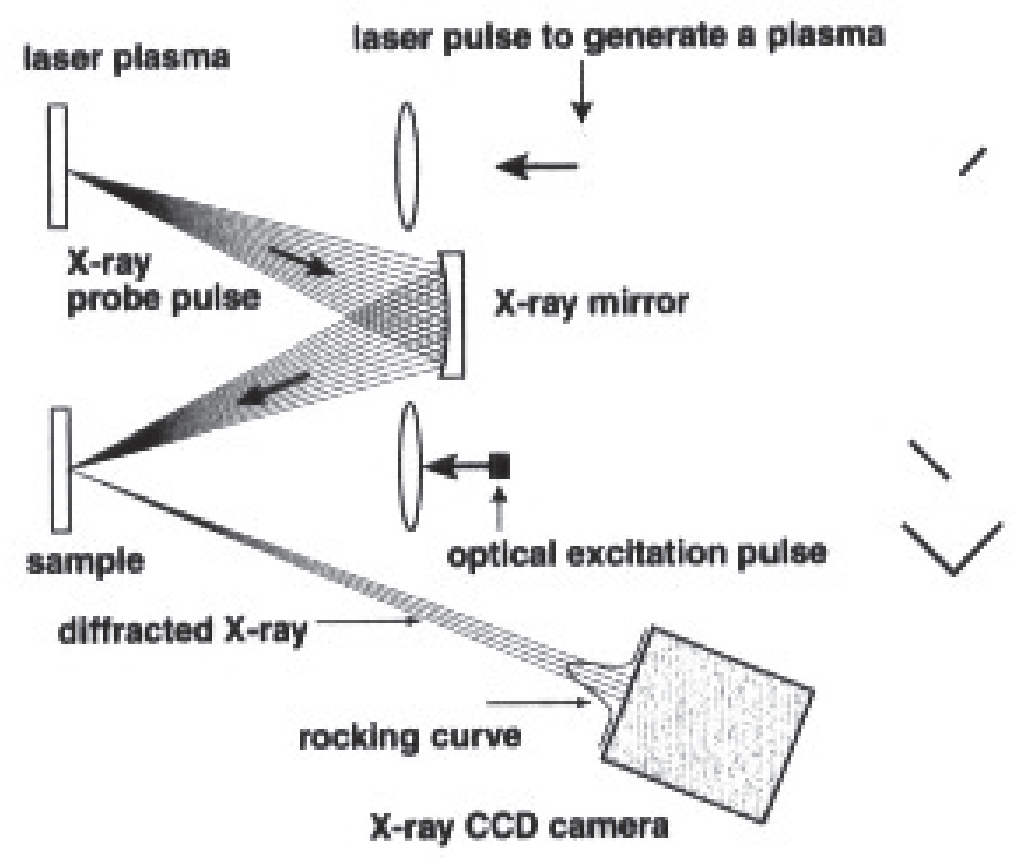

Fig. 6. Schematic of an optical pump- X-ray probe experiment [42].

One of the advantages of using X-ray beams for spectroscopic aims is the deeper penetration of coherent X-ray beam into the material if the wavelength is less or in the order of lattice 
spacing. Under these conditions X-ray diffraction would be strongly dominated by the bulk crystal ignoring the damaged or melted subsurface layers.

In such a way, different X-ray spectroscopical techniques have been developed as X-ray absorption spectroscopy (XAS), extended X-ray absorption fine structure spectroscopy (EXAFS) absorption near edge spectroscopy (XANES) inelastic X-ray Raman scattering (XRS), and X-ray emission spectroscopy (XES).

As an example, we demonstrate the concept of XRS (Fig. 7.).
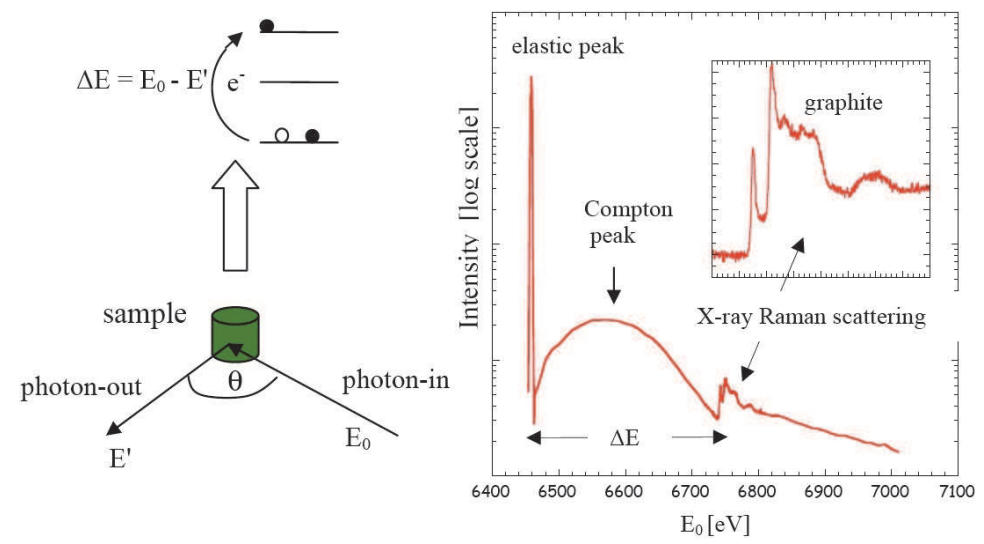

Fig. 7. Left: Concept of XRS. The energy transfer from an inelastically scattered photon results in the excitation of a core electron into an empty state. Right: Complete scattering spectrum from graphite. Intensity versus incident energy $\mathrm{E}_{0}$ is plotted, analyzer energy $\mathrm{E}^{\prime}$ is fixed at $6460 \mathrm{eV}$ [41].

Concerning the instrumentation different kinds of wavelength dispersive devices are in utilizations for spectroscopical applications e.g., cylindrically curved analyzers and position sensitive detectors (PSD) (see Fig. 8)

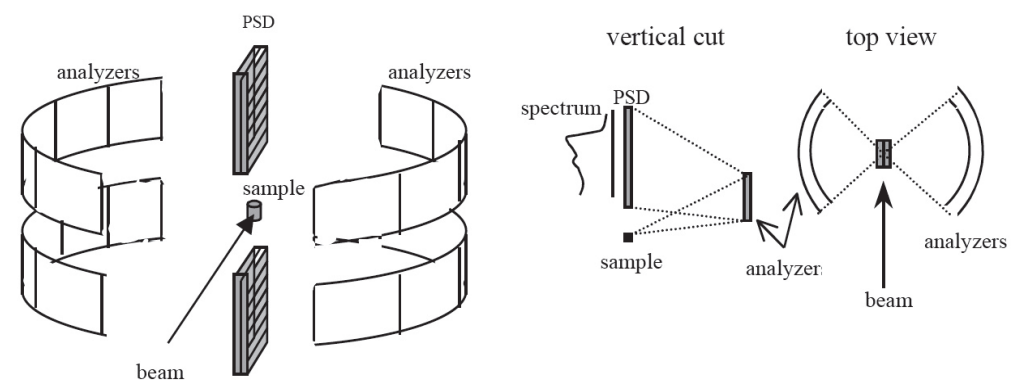

Fig. 8. Schematic setup four arrays of cylindrically curved crystals in sagital focusing mode. Scattering of a point source beam is analyzed at different energies (see vertical cut) resulting in a spectrum on the PSDs. For XRS the setup is rotated by $90^{\circ}$ for scattering in the predominantly vertical plane [41].

As a sample of nice characteristic results of XANES/EXAFS we turn to Fig. 9. 


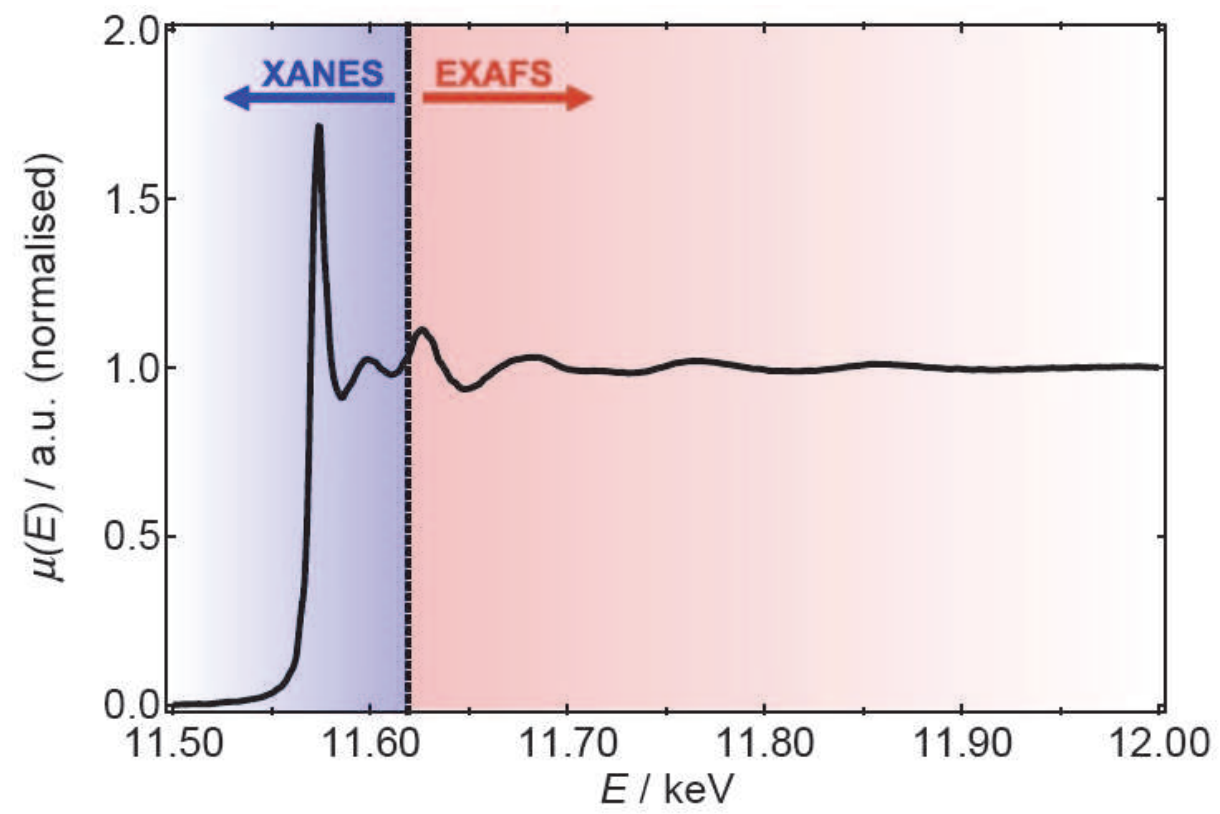

Fig. 9. XAS spectrum of a molecule (PtPOP) in solution illustrating the two regions: the lowenergy XANES region up to $\sim 50 \mathrm{eV}$ above the IP and the high-energy EXAFS region $>50 \mathrm{eV}$. The spectrum has been normalized.

\section{Time resolved $\mathrm{THz}$ spectroscopy}

In the optical wavelength scale the $\mathrm{THz}$ region makes a bridge between microwaves and infrared domains. This is located at about $10^{12} \mathrm{~Hz}$, so called terahertz region. Because of the quite low phonon energies in this region, the terahertz spectroscopy mainly is devoted to carry investigations in the exploration of infraband/subband excitations (transition). The Trays are harmless for the human body; therefore, one can find applications in basic medical research and security [34].

The materials used for generation of terahertz radiation by optical rectification can also be used for its detection by using the Pockels effect where certain crystalline materials become birefringent in the presence of an electric field. The birefringence caused by the electric field of a terahertz pulse leads to a change in the optical polarization of the detection pulse, proportional to the terahertz electric-field strength. With the help of polarizers and photodiodes, this polarization change can be measured. 

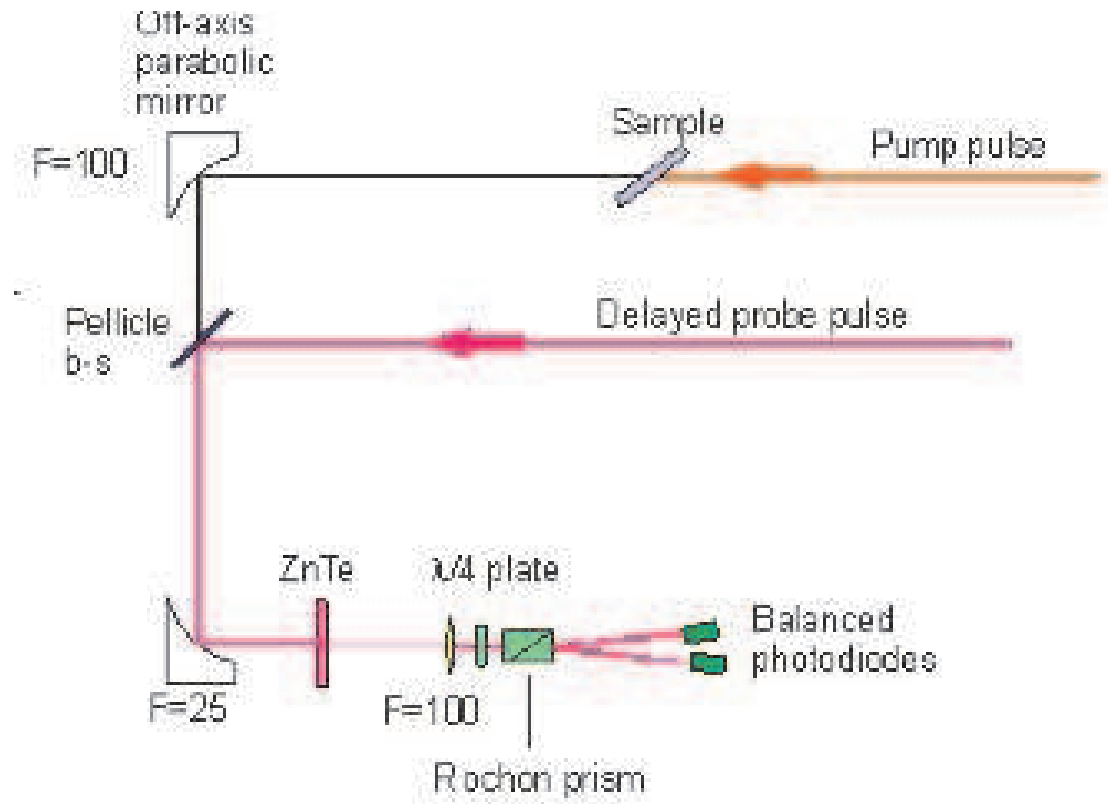

Fig. 10. Setup for the measuring of polarization change

A typical setup for transmission THz spectroscopy is shown in Fig. 11.

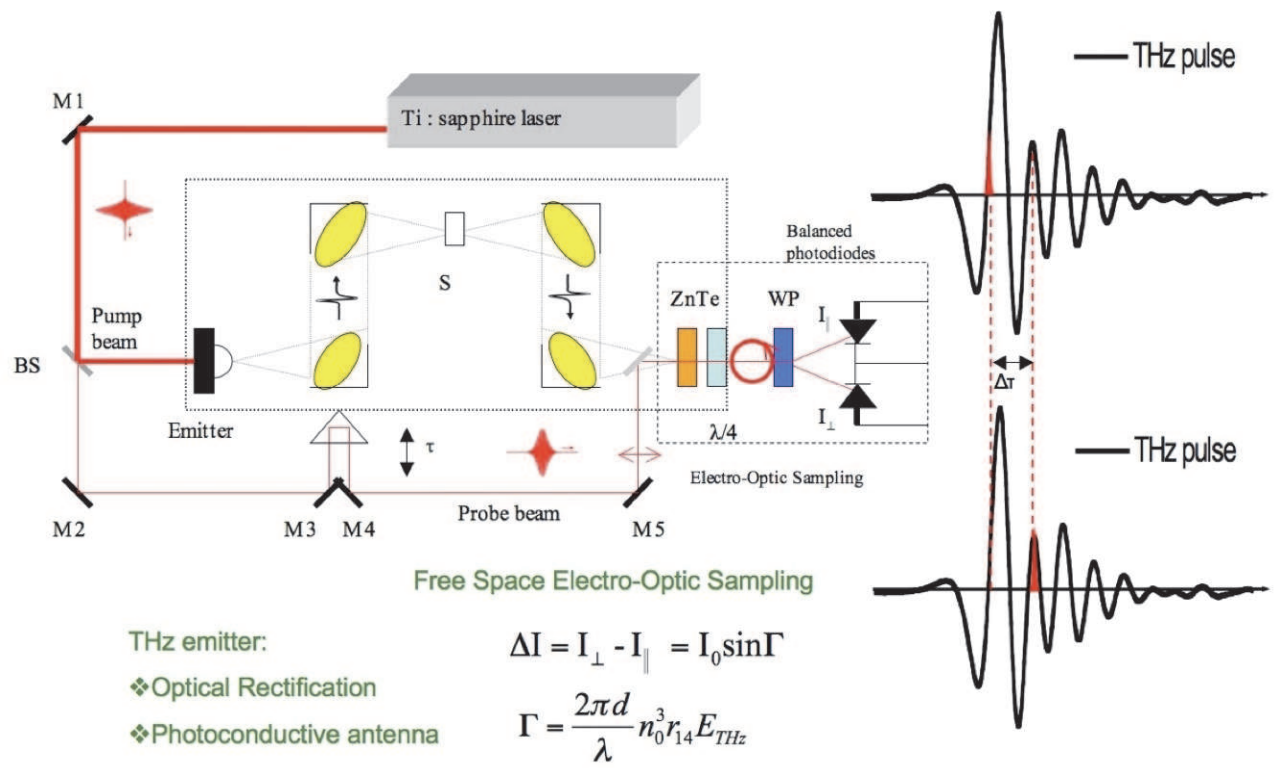

Fig. 11. A typical setup for transmission $\mathrm{THz}$ spectroscopy 
As a nice example of $\mathrm{THz}$ transient conductivity spectrum we do example of Si measured and fitted by Drude model (Fig. 12) [37].

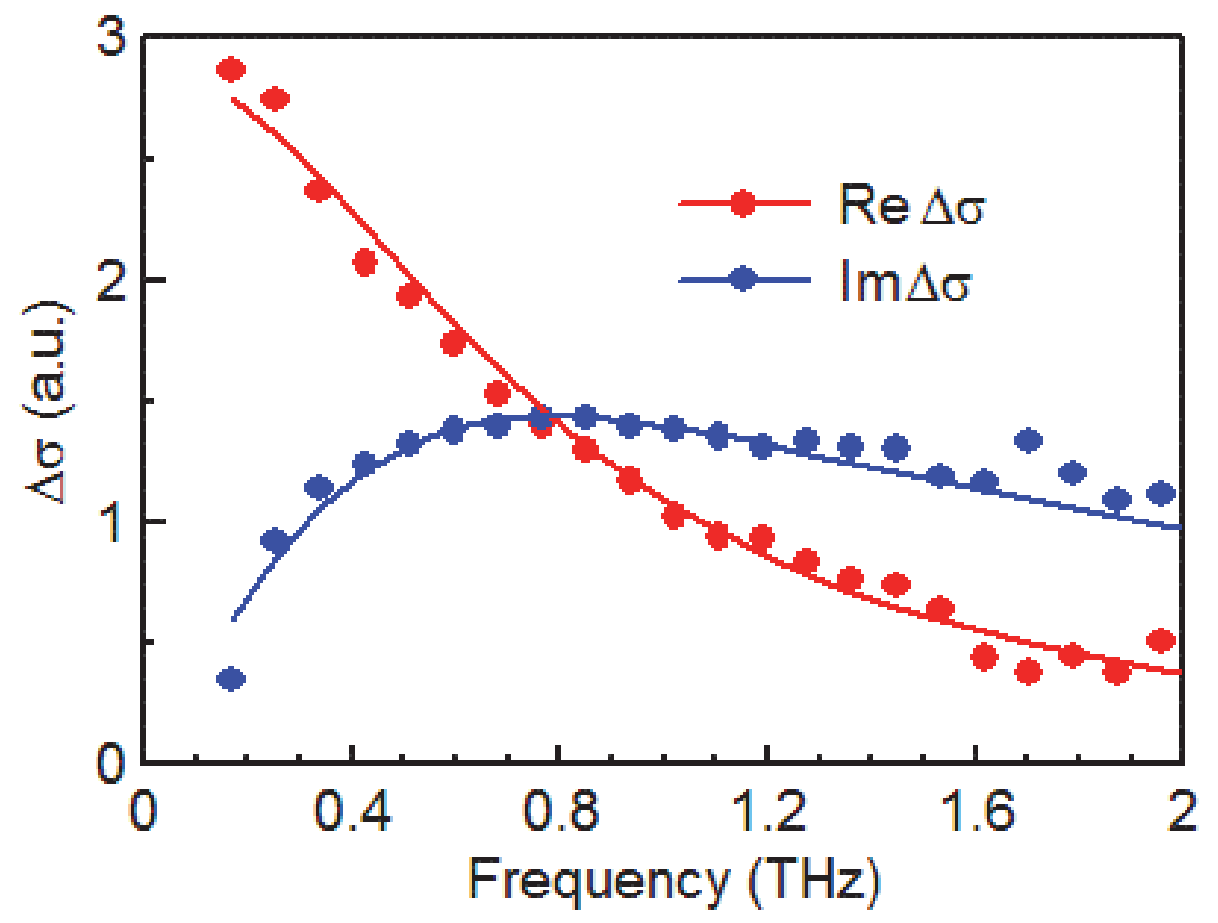

Fig. 12. Variation of conductivity in Si [37]

\section{Ultrafast infrared spectroscopy}

In the infrared spectral region (1-25 $\mu \mathrm{m}$ wavelengths) the consequences of Heisenberg uncertainty principle are especially significant, e.g., for a $200 \mathrm{fs}$ pulse duration we get spectral bandwidth about $75 \mathrm{~cm}^{-1}$.

For investigations of large and complex molecules the use of visible spectrum is not so convenient because of the overlapping features due to broad spectrum one can get only a few structural information. Therefore, to get useful information with high temporal resolution and sensitivity with proper reliability, we usually use combined (visible and IR) pump and probe variations [38]. 
As an example of a possible IR ultrashort arrangement, we show a scheme of experimental equipment devoted to examine charge-carrier dynamics in a polymer (PoV) (Fig. 13).
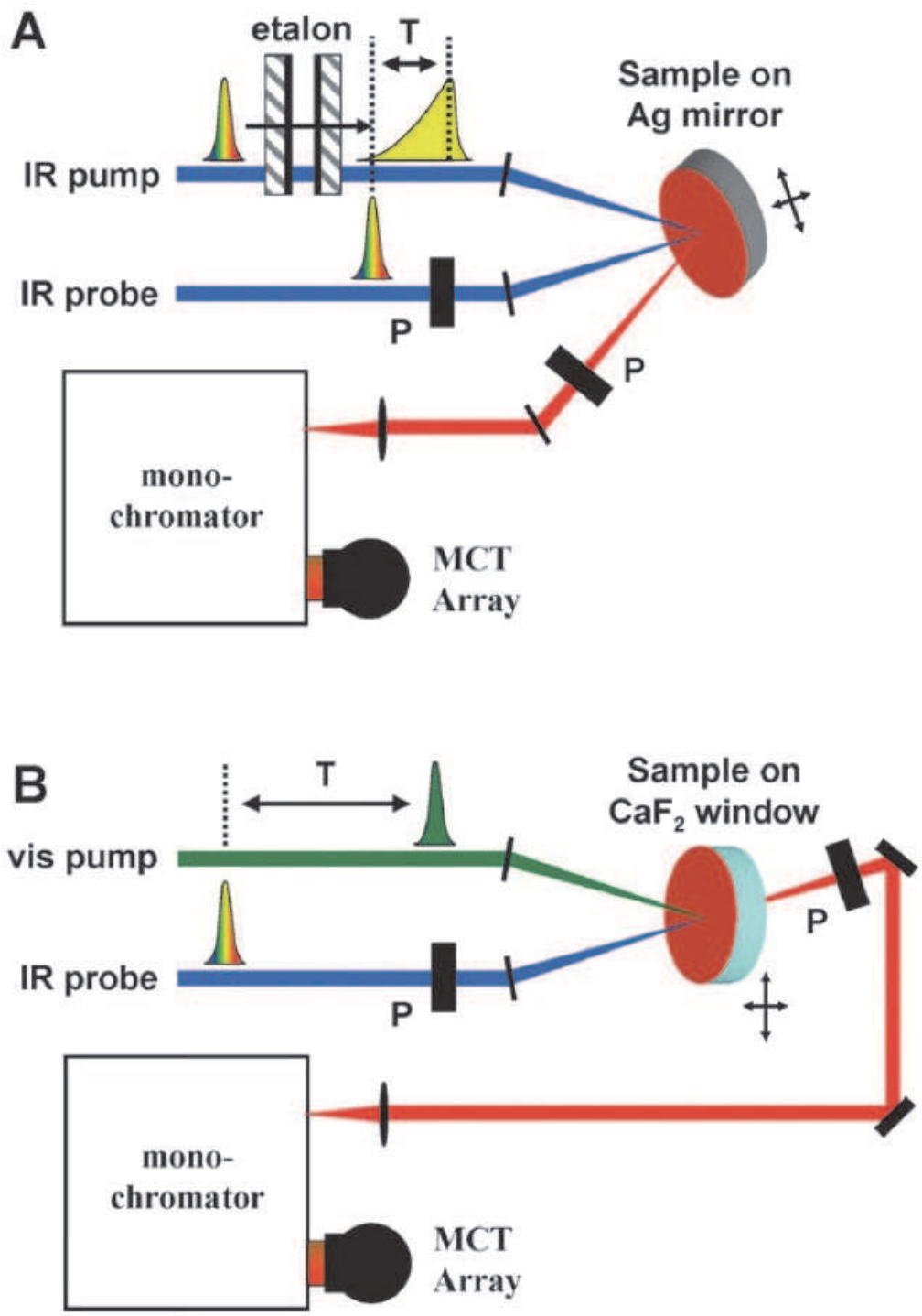

Fig. 13. Diagrams of the beam and sample geometries used for (A) 2D IR and (B) visible pump IR probe experiments. These geometries eliminate non-resonant signals that frequently interfere with ultrafast vibrational spectroscopic studies. The symbols represent: etalon - tunable Fabry-Perot interference filter, $\mathrm{T}$ - optical delay between the pump and probe pulses, $\mathrm{P}$-polarizer, MCT Array - mercury cadmium telluride multichannel infrared array detector [33]. 
With the help of continuous wave IR source is used as a probe beam. Passing through the sample this probe beam is overlapped with fs pump beam. The time resolution is determined by applying a second fs visible pulse to gate the probe beam by up conversion. As a result of interaction we get a pulse with a sum frequency of IR and visible beams while the intensity will be related to the IR absorption.

The temporal delay changing of up conversion one can swap the dynamics of the event. Moreover due to such a up conversion linear detectors in visible are used with high sensitivity and resolution. The CW IR source usually have a very narrow line width, therefore the resolution depends only on homogeneous and inhomogeneous broadening caused by the sample itself.

There is another method avoiding the consequences of Heisenberg principle based on using a short IR pulse to be passed through the sample. After that this beam is dispersed in a monochromator to raise the frequency resolution [40].

In such a realization, the short pulse causes polarization field in the sample. As a polarization a coherent radiation emerges with the probe beam. Therefore, the resolution will be limited with the interaction time of the field with the sample (optical dephasing).

The ultrafast infrared spectroscopy proved to be an excellent tool to carry out structural and dynamical investigations in different areas of chemistry, biophysics, and organic chemistry, especially in the diagnostic of transient states. As an example of the dynamics of dissociation of ICN molecule is shown in Fig. 14.

\section{Gain Clamping}
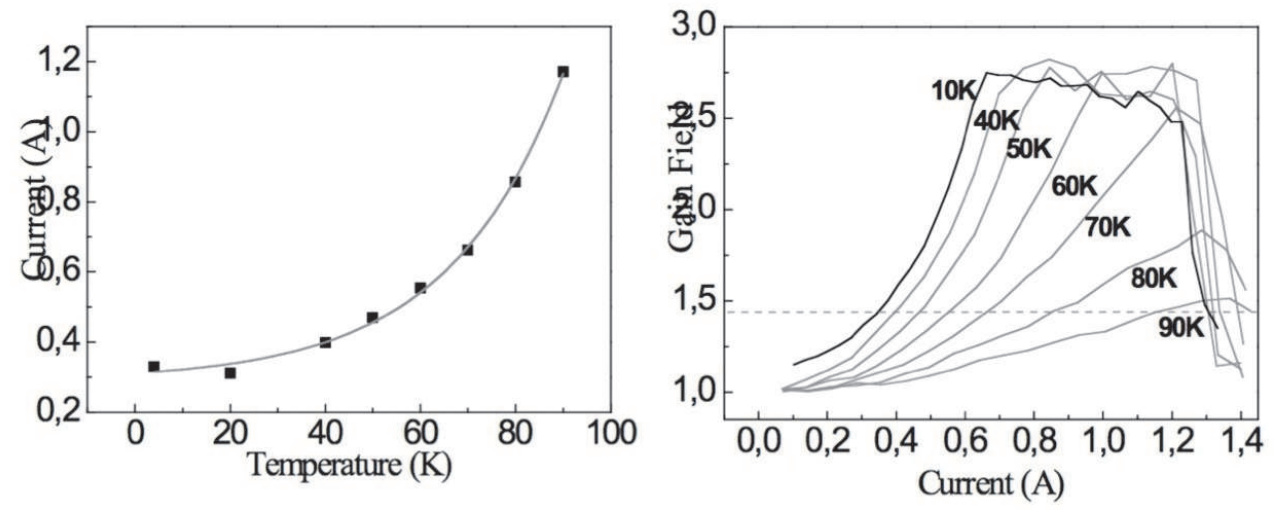

Fig. 14. Dissociation of ICN molecule

\section{Some results of ultrashort spectroscopy methods' applications in different fields of material science}

- Observations of ultrafast phase transitions 
It is well known that $\mathrm{VO}_{2}$ at moderate temperature $\mathrm{T}>67{ }^{\circ} \mathrm{C}$ goes through a phase transformation from an insulating to a metallic phase [41-43].

The conductivity changes dramatically ( about $10^{5}$ fold) while the crystal structure changes from monoclinic to rutile.

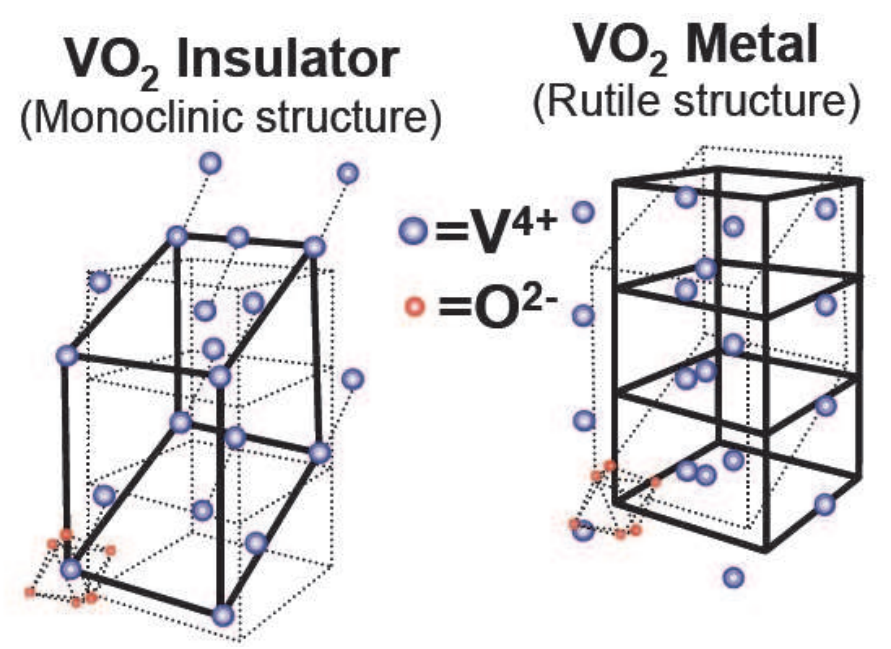

Fig. 15. The "structural bottleneck" in the transition arises from the time needed for the vanadium atoms to change from the monoclinic structure associated with the insulator to the rutile structure of the metal. [44].

The difference in a reflectivity of the structures is also dominant.

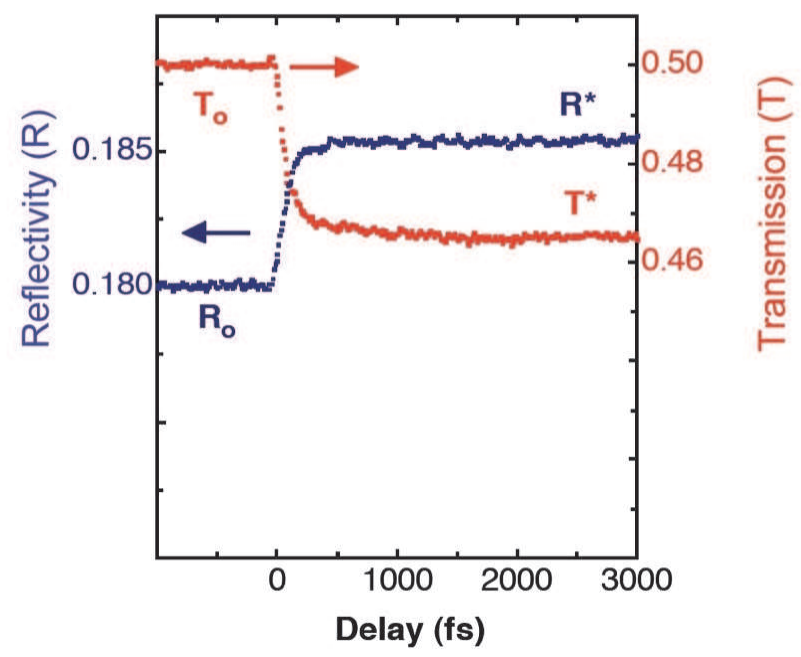

Fig. 16. The reflectivity of the metallic state is higher than that of the insulating state; use of an ultrafast probe pulse allows the measurement of the transition time with $15 \mathrm{fs}$ resolution ( $1 \mathrm{fs}=10^{-15}$ second $)$ [44]. 
The dynamics of the changes of electron correlation and so far reflectivities was studied with pump and probe spectroscopic methods; using ultrashort X-ray spectroscopy. The time transition limit for phase transformation from monoclinic to rutile was found about $80 \mathrm{fs}$ [44].

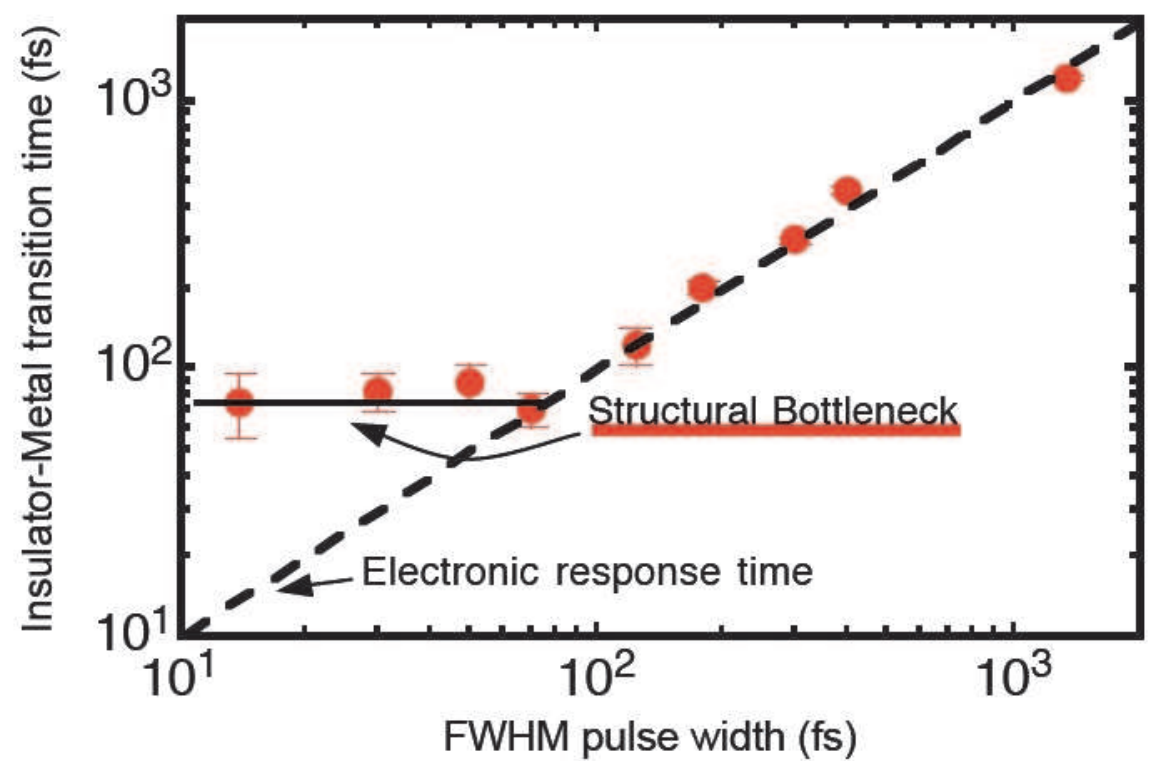

Fig. 17. The observed transition time for the insulator-metal transition (red data points) becomes progressively shorter with pulse duration down to $80 \mathrm{fs}$, at which point a fundamental limit is imposed by the material properties [44].

Direct band-to-band recombination in silicon nanocrystals and porous silicon

The silicon-bulk-crystal has interband optical transitions only with the participations of phonons (to evaluate the energy and momentum conservation laws). The reduction of size of silicon crystal e.g. nanosized or porous silicon show zero-phonon optical transitions which is promising for fabrication of light emitting devices for Si microelectronics. [44] To demonstrate the existence of such a transition and to get data for dynamics of the different ultrafast spectroscopic methods had been used such as time resolved photoluminescence, pump and probe phonon echoes and for wave mixing.

\section{References}

[1] László Nánai, Zsolt Benkő, Renat R. Letfullin and Thomas F. George, Laser-Matter Interactions: Nanostructures, Fabrication and Characterization in T. F. George, D. Jelski, R. R. Letfullin and G. P. Zhang, Editors, Computational Studies of New Materials II: From Ultrafast Processes and Nanostructures to Optoelectronics, Energy Storage and Nanomedicine (World Scientific, Singapore, 2011), 540 pages.

[2] Trebino R., Frequency-resolved opticalgratings: The measurements of ultrashort pulses, Kluwer publisher 2002 
[3] DeLong, K. W., R. Trebino, J. Hunter, and W. E. White, J. Opt. Soc. Amer. B, v. 11, p. 2206-2215 (1994).

[4] Nánai L., Szatmári S., Int. Conf. of Condensed. Matter. Mugla (Turkey) 26-28 May (2008) p. 27

[5] M. Dantus, P. Gross, Encyclopedia of Applied Physics 22, 431-456 (1998)

[6] Workman Jerry Jr., Springsteen Art, Applied Spectroscopy: A Compact Reference for Practitioners, Academic Press 1998.

[7] M. Cardona, R. Merlin, Light Scattering in Solids topics in Applied Physics, vol. 108 Springer 2007

[8] Rickard M. A., Pakoulev A. V., Mathew N. A., et al., J. Phys. Chem. A, 111, 1163-1166 (2007).

[9] Tranter G.E., Holmes J.L., Lindon J.C., Editors, Encyclopedia of Spectroscopy and Spectrometry, Volumes 1-3, Academic Press 2000

[10] Duckett S., Gilbert B., Foundations of Spectroscopy, Volumes 1-3, Oxford University Press 2000.

[11] Demtröder Wolfgang, Laser Spectroscopy, Volumes 1-3, 3rd Edition, Springer 2002.

[12] T. Elsaesser, M. Woerner, Physics Reports, 321 (1999) 253-305

[13] Cavalieri A. L. et al, Attosecond time-resolved spectroscopy at surfaces in Dynamics at Solid Surfaces and Interfaces, ed. By P. Hvroje and M. Wolf WILEY VCH 2010

[14] D. R. Brian, Science, 253/5078 p.1913

[15] Hörlein R., Thesis, Investigation of the XUV Emission from the Interaction of Intense Femtosecond Laser Pulses with Solid TargetsMünchen University 2008

[16] P.B. Allen, Phys. Rev. Lett. 59, 1460 (1987).

[17] M.I. Kaganov, I.M. Lifshitz, L.V.Tantarov, Zh. Exsp. Theor. Fiz. 31, 232 (1956) [Sov. Phys. JETP 4, 173 (1957)]

[18] Reid D. T., Measuring ultrafast laser pulses in Ultrafast photonics ed. By A. Miller, D. T. Reid. M Finlayson, Taylor and Francis 2004

[19] F. Krausz, Phys. World 14, 41 (2004)

[20] M. Schultze and al, Journal of electron spectroscopy and related phenomena (2011) doi:10.1016/j.elspec.2011.01003

[21] R.L. Samad et al., Ultrashort Laser Pulses Applications, IPEN-CNN/SP Brasil

[22] Krausz, F. \& Ivanov, M. (2009). Attosecond physics. Rev. Mod. Phys., 81, 1, 163-234, ISSN: 0034-6861

[23] R. Paschotta, Encyclopedia of laser physics and technology Wiley VCH (WWW.virtualLibrary)

[24] Rick Trebino lectures at https://www.physics.gatech.edu/user/rick-trebino

[25] R. Hoffmann American Scientist, 87, 308-311 (1999)

[26] A. H Zewail et al, Journal of physical chemistry, 100:12701-12724. (1996)

[27] Reiter E. et al, PRL, 105243902 (2010)

[28] Cavalieri, A. L. et al., Attosecond spectroscopy in condensed matter. Nature 449,1029 (2007).

[29] M. Dohle et al, Berichte der Bunsengesellschaft für physikalische Chemie Volume 99, Issue 3, pages 478-484, März 1995

[30] V. Schmidt, W. Husinsky*, G. Betz Applied Surface Science, 197-198 (2002) 145-155 
[31] Diels J. C. W. Rudolph, Ultrafast laser pulse phenomena: fundamentals, techniques and applications on femtosecond time scale, Academic Press Burlington MA USA 2006

[31]Uwe Bergmann, LOI for proposal „Wavelength Dispersive Optics for Ultrafast X-ray Spectroscopy at LCLS" Stanford Synchrotron Radiation Laboratory Menlo Park, California 94025

[32] Renske Marjan van der Veen THÈSE NO 4905 (2010) Ultrafast X-ray and Optical Spectroscopy of Binuclear Molecular Complexes, Lausanne 2010.

[33] Pensack R.D et al. Phys. Chem. Chem. Phys 2009, 11, 2575 - 2591

[34] file:///F:/InTech/44_Carito_Time-resolved\%20terahertz\%20spectroscopy.htm

[35] Han-Kwang Nienhuys, and Villy Sundström, Appl. Phys. Lett. 87, 012101 (2005)

[36] Han-Kwang Nienhuys, and Villy Sundström, Phys. Rev. B 71, 235110 (2005)

[37] Hoffmann M C., Yeh K. L., Hebling J., Nelson K A., Optics Express 15(18) 11706-13 (2007)

[38] Jedju T. M. et al, Appl. Opt., 312684 (1992)

[39] Fecko J.C., Science 2003, Vol. 301 no. 5640 pp. 1698-1702

[40] Szekerle J.P. et al, J. Chem. Phys. 95, 5403 (1991)

[41] Berkeley Lab research Highlight by Schoenlein R., “Ultrafast phase transitions” U. S. Dept of Energy Divison of Material Science

[42] Cavalieri A. et al, Phys rev B Rapid Comm., 70, 161102 (2004)

[43] Cavalieri A. et al, Phys Rev Lett., 95, 067405 (2005)

[44] Wen X., Ultrafast Spectroscopy of Semiconductor Nanostructures, PhD Thesis, Swinburne Univ. Melbourne, Australia, 2007 


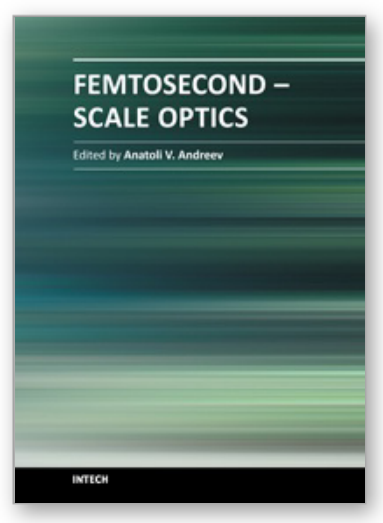

\author{
Femtosecond-Scale Optics \\ Edited by Prof. Anatoly Andreev
}

ISBN 978-953-307-769-7

Hard cover, 434 pages

Publisher InTech

Published online 14, November, 2011

Published in print edition November, 2011

With progress in ultrashort ultraintense laser technologies the peak power of a laser pulse increases year by year. These new instruments accessible to a large community of researchers revolutionized experiments in nonlinear optics because when laser pulse intensity exceeds or even approaches intra-atomic field strength the new physical picture of light-matter interaction appears. Laser radiation is efficiently transformed into fluxes of charged or neutral particles and the very wide band of electromagnetic emission (from $\mathrm{THz}$ up to $\mathrm{x}$-rays) is observed. The traditional phenomena of nonlinear optics as harmonic generation, self-focusing, ionization, etc, demonstrate the drastically different dependency on the laser pulse intensity in contrast the well known rules. This field of researches is in rapid progress now. The presented papers provide a description of recent developments and original results obtained by authors in some specific areas of this very wide scientific field. We hope that the Volume will be of interest for those specialized in the subject of laser-matter interactions.

\title{
How to reference
}

In order to correctly reference this scholarly work, feel free to copy and paste the following:

László Nánai, Szabolcs Beke and Koji Sugioka (2011). Ultrafast Time-Resolved Spectroscopy, FemtosecondScale Optics, Prof. Anatoly Andreev (Ed.), ISBN: 978-953-307-769-7, InTech, Available from: http://www.intechopen.com/books/femtosecond-scale-optics/ultrafast-time-resolved-spectroscopy

\section{INTECH}

open science | open minds

\author{
InTech Europe \\ University Campus STeP Ri \\ Slavka Krautzeka 83/A \\ 51000 Rijeka, Croatia \\ Phone: +385 (51) 770447 \\ Fax: +385 (51) 686166 \\ www.intechopen.com
}

\author{
InTech China \\ Unit 405, Office Block, Hotel Equatorial Shanghai \\ No.65, Yan An Road (West), Shanghai, 200040, China \\ 中国上海市延安西路65号上海国际贵都大饭店办公楼405单元 \\ Phone: +86-21-62489820 \\ Fax: $+86-21-62489821$
}


(C) 2011 The Author(s). Licensee IntechOpen. This is an open access article distributed under the terms of the Creative Commons Attribution 3.0 License, which permits unrestricted use, distribution, and reproduction in any medium, provided the original work is properly cited. 